\title{
TUMOR BUDDING Y EL CÁNCER COLORRECTAL*
}

\author{
Drs. María Labalde M. ${ }^{1}$, Francisco Javier Labrador V. ${ }^{1}$, Arturo Golitsin D. ${ }^{1}$, \\ Santiago Pinto G. ${ }^{1}$, Jorge Carlin G. ${ }^{1}$, Jorge García-Parreño J. ${ }^{1}$ \\ 1 Cirugía General y del Aparato Digestivo. Hospital Universitario de Guadalajara. \\ Guadalajara, España.
}

\begin{abstract}
Budding tumour and colorectal cancer

Introduction: Tumour budding (TB) is defined as the presence of clusters of tumoural cells detaching from invasive margin of main tumor. It is an independent adverse prognostic factor in colorectal cancer. The aim of this study is to determinate if severity of tumor budding is associated with others prognostic factors in colorectal cancer. Materials and Methods: The study group is composed by 43 patients ( 27 males and 16 females; average age 73.4 years, (27-91) with colorectal cancer who underwent curative surgery. The histologic method of tumour budding used in this study was described by Nakamura. The applied statistical software was G-Stat 2.0. Statistical significance is accepted at $\mathrm{p}<0.05$. Results: High grade of TB was significantly associated with lymph node metastasis $(\mathrm{p}=0.027)$, infiltrative tumour-border configuration $(\mathrm{p}=0.016)$, lymphvessels invasion $(p=0.02)$, perineural invasion $(p=0.009)$ and tumor deposits $(p=0.018)$. There was a significant association with low grade of TB and peritumoural lymphocytic infiltration $(p=0.004)$. Conclusions: High grade of TB is significantly associated with other adverse prognostic factors as lymph node metastasis, infiltrative tumour-border configuration, lymphvessels invasion, venous invasion, perineural invasion and tumor deposits; and low grade of TB with favorable prognostic factor as peritumoural lymphocytic infiltration in colorectal cancer. Tumour budding can help to identify hig-risk patients with colorectal cancer.

Key words: Budding tumour, colorectal cancer, prognostic factor.
\end{abstract}

\section{Resumen}

Introducción: El tumor budding $(T B)$ es la presencia de células tumorales aisladas o en pequeños grupos situadas en el frente infiltrante del tumor. Su hallazgo en alto grado es un factor de mal pronóstico independiente del cáncer colorrectal. El objetivo de este trabajo es determinar si el grado de TB está asociado con otros factores pronósticos del cáncer colorrectal. Materiales y Métodos: Se analizaron retrospectivamente 43 pacientes (27 varones y 16 mujeres) con una edad media de 73,4 años (27-91) intervenidos por cáncer colorrectal. El método histológico utilizado para determinar la presencia de TB fue el descrito por Nakamura en 2005. El análisis estadístico se realizó con el programa G-Stat2.0. Las diferencias se consideraron significativas si $\mathrm{p}<0,05$. Resultados: La presencia de TB de alto grado se asocia

* Recibido el 3 de abril de 2014 y aceptado para publicación el 12 de mayo de 2014.

Los autores no refieren conflictos de interés.

Correspondencia: Dra. María Labalde M. marialm007@hotmail.com 
significativamente con la presencia de metástasis a ganglios linfáticos $(\mathrm{p}=0,027)$, patrón de crecimiento infiltrativo $(\mathrm{p}=0,016)$, invasión linfática $(\mathrm{p}=0,02)$, perineural $(\mathrm{p}=0,009)$ y depósitos tumorales discontinuos $(\mathrm{p}=0,018)$. El TB de bajo grado se relaciona con la presencia de reacción linfocitaria peritumoral $(\mathrm{p}=0,004)$. Conclusiones: El tumor budding alto grado se asocia con otros factores de mal pronóstico, como metástasis a ganglios linfáticos, crecimiento infiltrativo, invasión linfática, perineural y depósitos tumorales discontinuos; y el tumor budding bajo grado con factores de buen pronóstico del cáncer colorrectal como reacción linfocitaria peritumoral. El análisis del grado de tumor budding podría ayudar a identificar a pacientes de peor pronóstico con cáncer colorrectal.

Palabras clave: Tumor budding, cáncer colorrectal, factor pronóstico.

\section{Introducción}

El cáncer colorrectal es la tercera neoplasia más frecuente en el mundo y supone la cuarta causa de muerte por cáncer ${ }^{1}$. El estudio de las características histopatológicas del tumor define el momento evolutivo del cáncer y orienta el pronóstico de la enferme$\mathrm{dad}^{2}$. La estadificación tumoral del American Joint Committe on Cancer/International Union against Cancer (AJCC/UICC) del $20107^{\mathrm{a}}$ edición incluye el sistema TNM (tumour-node-metastasis), la invasión venosa, linfática y perineural y la presencia de depósitos tumorales discontinuos y es el factor pronóstico actual más importante para los pacientes con $\mathrm{CCR}^{3}$. La valoración de otros factores pronósticos como el grado histológico, configuración del borde del tumor, tumor budding, tipo medular, niveles de antígeno carcinoembrionario, obstrucción intestinal y perforación del tumor durante la cirugía puede orientar el pronóstico del paciente así como el tratamiento de la enfermedad ${ }^{4}$.

El hallazgo histológico de budding tumor o gemación focal, definido como la presencia de células tumorales aisladas o en pequeños grupos de más de 5 células situadas en el frente infiltrante del tumor, es un factor de mal pronóstico independiente del $\mathrm{CCR}^{5-7}$, ya que estas células actúan a modo de avanzadilla preparando el terreno para que el frente invasor progrese ${ }^{8}$. Su hallazgo en alto grado está asociado con otras características de los tumores agresivos como el nivel $\mathrm{T}$ de invasión tumoral avanzado, metástasis ganglionares, grado bajo de diferenciación, patrón de crecimiento infiltrativo e invasión linfática ${ }^{9-11}$.

Los objetivos de este trabajo son determinar si la presencia de TB está asociada con otros factores anatomopatológicos de mal pronóstico del CCR como la afectación ganglionar, tamaño tumoral superior a $5 \mathrm{~cm}$, configuración del margen tumoral infiltrativa, invasión linfática, vascular y perineural y existencia de depósitos tumorales discontinuos; y estudiar su relación con factores de buen pronóstico del CCR como la presencia de reacción linfocitaria intratumoral y peritumoral e inestabilidad de los microsatélites.

\section{Material y Método}

Se analizó retrospectivamente un total de 43 pacientes con CCR sometidos a cirugía en el Hospital Universitario de Guadalajara durante el período comprendido desde abril a noviembre de 2013, de los que $27(63 \%)$ fueron varones y $16(37 \%)$ mujeres, con una edad media de 73,7 años (27-91). Se excluyeron los casos en los que no se realizó resección del tumor. El diagnóstico histológico de la biopsia del tumor obtenida mediante colonoscopia fue de adenocarcinoma en todos los casos. La localización del tumor fue en el colon derecho en $16(37 \%)$ casos, izquierdo en $16(17 \%)$ y recto en 11 (26\%). La distribución del riesgo anestésico según la escala ASA fue: 2 (5\%) pacientes con ASA I, 24 (55\%) con ASA II, $15(35 \%)$ con ASA III y $2(5 \%)$ con ASA IV. Se realizaron $13(30 \%)$ hemicolectomías derechas, 13 (30\%) sigmoidectomías, 8 (18\%) resecciones anteriores bajas, $3(7 \%)$ resecciones segmentarias de colon, $3(7 \%)$ hemicolectomías izquierdas, $2(5 \%)$ intervenciones de Hartmann y una (3\%) amputación abdominoperineal. Respecto a los estadios tumorales según la clasificación TNM del AJCC 7 $7^{\mathrm{a}}$ ed. 2010 se incluyeron 20 (46\%) casos con estadio II, 16 (37\%) con estadio III, 5 (12\%) con estadio IV y 2 con estadio I ( $5 \%$ ).

El estudio histológico de los especímenes resecados sigue las recomendaciones de la European Guidelines for Quality Assurance in Colorectal Screening and Diagnosis $1^{a}$ ed. ${ }^{12}$. Se recogieron datos de los informes de anatomía patológica de las muestras examinadas por un patólogo sobre tumor budding, tipo y grado histológico, estadificación según TNM ( $7^{\mathrm{a}}$ edición), número de ganglios linfáticos aislados, patrón de crecimiento, invasión linfática, vascular y perineural, depósitos tumorales discontinuos, tamaño tumoral, diferenciación mucinosa, reacción linfocitaria intratumoral y peritumoral e inestabilidad de los microsatélites.

El método anatomopatológico utilizado en este estudio que determina la presencia de TB y su grado es el descrito y validado por Nakamura en $2005^{13,14}$. Emplea la tinción Hematoxilina-eosina sobre cortes de tumor que incluyen las secciones 
más extensas del borde invasor. Su examen bajo el microscopio óptico permite identificar grupos de células indiferenciadas malignas en el frente invasor que constituyen la desdiferenciación focal o TB. Se definen cuatro niveles: ausente (no hay células indiferenciadas más allá del margen invasor), leve (desdiferenciación focal presente en menos de 1/3 de la longitud del margen invasor), moderado (entre $1 / 3$ y $2 / 3$ ), marcado (en más de $2 / 3$ ). Estos niveles pueden agruparse en dos grados: bajo (ausente y leve) y alto (moderado y marcado).

El análisis estadístico de los datos se realizó con el programa informático G-Stat 2.0. En el análisis descriptivo los resultados se expresaron como frecuencias (porcentajes) para variables cualitativas y como media (rango) para variables cuantitativas. Para comparar las variables cualitativas se aplicó la prueba de contraste de hipótesis $\chi^{2}$ y de Fisher, en aquellos casos en los que no se cumplen los criterios para utilizar $\chi^{2}$. Se consideraron las diferencias significativas cuando $\mathrm{p}<0,05$.

\section{Resultados}

\section{Análisis descriptivo de los grados de tumor budding}

La distribución de los grados de TB según la clasificación de Nakamura ${ }^{13}$ fue la siguiente: 32 (74\%) casos de bajo grado, entre los que 27 (63\%) se definieron como leve y $5(11 \%)$ como ausente, y $11(26 \%)$ casos de alto grado, entre los que se clasificaron $2(4 \%)$ casos como moderado y $9(22 \%)$ como marcado.

\section{Análisis descriptivo de las características histopatológicas del tumor}

Todos los casos incluidos en este estudio fueron adenocarcinomas. La distribución de los casos según la estadificación TNM de la AJCC ( $\left.7^{\mathrm{a}} \mathrm{ed}, 2010\right)$ y otras características histológicas se describen en la Tabla 1.

\section{Relación entre grados de tumor budding y factores de mal pronóstico del CCR}

Se analizó si existe relación entre el grado de TB y otros factores de mal pronóstico del CCR (Tabla 2).

Se demostró que existe asociación estadísticamente significativa entre el grado alto de TB y otros factores del mal pronóstico del CCR incluidos en la estadificación TNM del AJCC ( $7^{\mathrm{a}}$ de., 2010) como la presencia de metástasis a ganglios linfáticos $(\mathrm{p}=0,027)$, invasión linfática $(\mathrm{p}=0,02)$, perineural $(\mathrm{p}=0,009)$ y depósitos tumorales discontinuos $(\mathrm{p}=0,018)$. Con respecto a otros factores del mal
Tabla 1. Análisis descriptivo de las características histopatológicas del tumor $(n=43)$

\begin{tabular}{|c|c|c|}
\hline & \multicolumn{2}{|c|}{ Frecuencia, n (\%) } \\
\hline Adenocarcinoma & \multicolumn{2}{|c|}{$43(100)$} \\
\hline $\begin{array}{l}\text { Grado histológico } \\
\text { Bajo } \\
\text { Alto }\end{array}$ & \multicolumn{2}{|c|}{$\begin{array}{r}38(88) \\
5(12)\end{array}$} \\
\hline $\begin{array}{l}\text { Estadificación TNM ( } 7^{\mathrm{a}} \text { ed., 2010) } \\
\text { - Nivel T invasión de la pared } \\
\text { - T1 } \\
\text { - T2 } \\
\text { - T3 } \\
\text { - T4 } \\
\text { - Afectación ganglionar } \\
\text { - N0 } \\
\text { - N1 } \\
\text { - N2 } \\
\text { - Metástasis a distancia } \\
\text { - M0 } \\
\text { - M1 }\end{array}$ & $\begin{array}{r}1 \\
4 \\
24 \\
14\end{array}$ & $\begin{array}{r}(2) \\
(9) \\
(56) \\
(33) \\
(56) \\
(28) \\
(16) \\
(84) \\
(16)\end{array}$ \\
\hline $\mathrm{n}$ de ganglios linfáticos aislados & \multicolumn{2}{|c|}{$18,3(10-39)$} \\
\hline Invasión linfática & 15 & $(35)$ \\
\hline Invasión vascular & 10 & $(23)$ \\
\hline Invasión perineural & 17 & $(40)$ \\
\hline Depósitos tumorales discontinuos & 11 & (26) \\
\hline $\begin{array}{l}\text { Patrón de crecimiento } \\
\text { Infiltrativo } \\
\text { Expansivo }\end{array}$ & $\begin{array}{l}26 \\
17\end{array}$ & $\begin{array}{l}(61) \\
(40)\end{array}$ \\
\hline Diferenciación mucinosa & 10 & $(23)$ \\
\hline Tamaño tumoral $>5 \mathrm{~cm}$ & 25 & $(58)$ \\
\hline Reacción linfocítica intratumoral & 20 & (46) \\
\hline Reacción linfocítica peritumoral & 34 & (79) \\
\hline IMS & 7 & (16) \\
\hline
\end{tabular}

pronóstico del CCR no incluidos en la estadificación TNM del AJCC ( $7^{\mathrm{a}}$ de., 2010) se ha encontrado asociación estadística entre el grado alto de TB y el crecimiento tumoral infiltrativo $(p=0,016)$. Sin embargo, no se ha encontrado significación estadística en la relación entre el grado alto de TB y la invasión venosa, el tamaño tumoral mayor o igual a $5 \mathrm{~cm}$, la diferenciación mucinosa y el grado histológico.

\section{Relación entre grados de tumor budding y factores de buen pronóstico del CCR}

Se analizó si existe relación entre el grado de TB y factores de buen pronóstico del CCR (Tabla 3 ).

Se demostró que existe asociación estadísticamente significativa entre el grado bajo de TB y la presencia de reacción linfocítica peritumoral 
Tabla 2. Relación entre grados de tumor budding y factores de mal pronóstico del CCR

\begin{tabular}{|c|c|c|c|c|}
\hline & & $\begin{array}{c}\text { TB alto } \\
\text { Grado (\%) } \\
\text { n }=11\end{array}$ & $\begin{array}{c}\text { TB bajo } \\
\text { Grado (\%) } \\
\text { n = 32 }\end{array}$ & Valor $\mathbf{p}$ \\
\hline Grado histológico & $\begin{array}{l}\text { Bajo } \\
\text { Alto }\end{array}$ & $\begin{array}{rr}9 & (21) \\
2 & (4)\end{array}$ & $\begin{array}{rr}29 & (67) \\
3 & (7)\end{array}$ & 0,589 \\
\hline Ganglios linfáticos regionales & $\begin{array}{l}\mathrm{N}+ \\
\mathrm{N} 0\end{array}$ & $\begin{array}{rr}8 & (18) \\
3 & (7)\end{array}$ & $\begin{array}{ll}11 & (26) \\
21 & (49)\end{array}$ & $0,027^{*}$ \\
\hline Invasión linfática & $\begin{array}{l}\text { Sí } \\
\text { No }\end{array}$ & $\begin{array}{rr}7 & (16) \\
4 & (9)\end{array}$ & $\begin{aligned} 8 & (19) \\
25 & (56)\end{aligned}$ & $0,02 *$ \\
\hline Invasión vascular & $\begin{array}{l}\text { Sí } \\
\text { No }\end{array}$ & $\begin{array}{ll}5 & (11) \\
6 & (15)\end{array}$ & $\begin{aligned} 5 & (11) \\
27 & (63)\end{aligned}$ & 0,091 \\
\hline Invasión perineural & $\begin{array}{l}\text { Sí } \\
\text { No }\end{array}$ & $\begin{array}{rr}8 & (19) \\
3 & (7)\end{array}$ & $\begin{aligned} 9 & (21) \\
23 & (53)\end{aligned}$ & $0,009^{*}$ \\
\hline Depósitos tumorales discontinuos & $\begin{array}{l}\text { Sí } \\
\text { No }\end{array}$ & $\begin{array}{ll}6 & (15) \\
5 & (11)\end{array}$ & $\begin{aligned} 5 & (11) \\
27 & (63)\end{aligned}$ & $0,018 *$ \\
\hline Patrón crecimiento & $\begin{array}{l}\text { Infiltrativo } \\
\text { Expansivo }\end{array}$ & $\begin{array}{rr}10 & (24) \\
1 & (2)\end{array}$ & $\begin{array}{ll}16 & (37) \\
16 & (37)\end{array}$ & $0,016^{*}$ \\
\hline Tamaño $>5 \mathrm{~cm}$ & $\begin{array}{l}\text { Sí } \\
\text { No }\end{array}$ & $\begin{array}{rr}7 & (17) \\
4 & (9)\end{array}$ & $\begin{array}{ll}18 & (42) \\
14 & (32)\end{array}$ & 0,668 \\
\hline Diferenciación mucinosa & $\begin{array}{l}\text { Sí } \\
\text { No }\end{array}$ & $\begin{array}{rr}7 & (17) \\
4 & (9)\end{array}$ & $\begin{array}{ll}22 & (51) \\
10 & (23)\end{array}$ & 0,754 \\
\hline
\end{tabular}

TB: tumor budding. *Diferencias estadísticamente significativas.

Tabla 3. Relación entre grados de tumor budding y factores de buen pronóstico del CCR

\begin{tabular}{|c|c|c|c|c|}
\hline & & $\begin{array}{c}\text { TV alto } \\
\text { Grado (\%) } \\
\mathbf{n}=11\end{array}$ & $\begin{array}{c}\text { TB bajo } \\
\text { Grado (\%) } \\
\text { n= } 32\end{array}$ & Valor $\mathbf{p}$ \\
\hline Reacción linfocítica peritumoral & $\begin{array}{l}\text { Sí } \\
\text { No }\end{array}$ & $\begin{array}{ll}5 & (12) \\
6 & (14)\end{array}$ & $\begin{array}{rr}29 & (67) \\
3 & (7)\end{array}$ & $0,004 *$ \\
\hline Reacción linfocítica intratumoral & $\begin{array}{l}\text { Sí } \\
\text { No }\end{array}$ & $\begin{array}{rr}7 & (16) \\
4 & (9)\end{array}$ & $\begin{array}{ll}13 & (30) \\
19 & (44)\end{array}$ & 0,186 \\
\hline Inestabilidad de los microsatélites & $\begin{array}{l}\text { Sí } \\
\text { No }\end{array}$ & $\begin{array}{rr}8 & (19) \\
2 & (5)\end{array}$ & $\begin{aligned} 27 & (64) \\
5 & (12)\end{aligned}$ & 1,0 \\
\hline
\end{tabular}

TB tumor budding, RL, IMS. *Diferencias estadísticamente significativas.

$(p=0,004)$. Sin embargo, no se ha encontrado significación estadística en la relación entre el grado bajo y la presencia de reacción intratumoral ni de inestabilidad de los microsatélites.

\section{Discusión}

El TB de alto grado es un factor de mal pronóstico independiente del cáncer colorrectal ${ }^{7,8}$ y también de otros tumores como el ampuloma ${ }^{15}$ y el cáncer de pulmón ${ }^{16}$. Nuestros resultados demuestran que existe relación estadísticamente significativa entre el tumor budding de alto grado y otros factores de mal pronóstico del cáncer colorrectal como la presencia de metástasis en ganglios linfáticos invadidos por tumor, invasión linfática y perineural, depósitos tumorales discontinuos y patrón de crecimiento infiltrativo. Estos resultados se asemejan a los de otros estudios ya que también relacionan el TB de alto grado con otros factores de mal pronóstico del cáncer colorrectal como el nivel $\mathrm{T}$ de invasión de la 
pared tumoral avanzado, grado bajo de diferenciación tumoral y metástasis a distancia ${ }^{17,18}$. Además, el presente artículo coincide con otras investigaciones en relacionar el TB de alto grado con metástasis ganglionares y con la presencia de invasión linfática ${ }^{19-21}$.

La estadificación tumoral del AJCC/UICC $7^{\mathrm{a}}$ ed. de 2010 en la que se incluye el sistema TNM (tumour-node-metastasis) y otros parámetros como la invasión linfática, venosa, perineural y la presencia de depósitos tumorales discontinuos, es el factor pronóstico más importante del cáncer colorrectal $^{3,22,23}$. Según esta clasificación la mayoría de los casos de CCR en estadio II no son seleccionados para quimioterapia adyuvante y la cirugía con resección $\mathrm{R} 0$ se considera el tratamiento curativo. Sin embargo, un porcentaje en torno al $25 \%$ de los estadios I-II con $\mathrm{N}$ negativos se comportan como estadios más avanzados y desarrollan metástasis a distancia $^{24,25}$. En pacientes con CCR en estadio II ( $\mathrm{N}$ negativos) las tasas de supervivencia a 5 años varían desde un $63 \%$, en casos con TB alto grado, hasta un $91 \%$, en casos de TB bajo grado ${ }^{26}$. El grado TB como factor de mal pronóstico independiente en el CCR puede ser útil para identificar ese subgrupo de pacientes en estadio II con peor pronóstico que se comportan como estadios más avanzados y que se beneficiarían de tratamientos adyuvantes tras la cirugía para aumentar las tasas de supervivencia ${ }^{27}$.

La presencia de TB intratumoral en las biopsias obtenidas mediante colonoscopia también se asocia a metástasis en ganglios linfáticos, estadio avanzado, invasión vascular y mal pronóstico ${ }^{28}$. De este modo, el estudio del grado de TB intratumoral en las biopsias de los estudios preoperatorios puede tener una gran relevancia clínica ya que podría contribuir a definir su pronóstico y planear la mejor estrategia terapéutica antes de la cirugía ${ }^{29}$.

Por otro lado, el TB de bajo grado se ha asociado a otro factor de buen pronóstico del CCR como la infiltración linfocítica peritumoral de forma estadísticamente significativa en este trabajo. Otras publicaciones también demuestran esta asociación ${ }^{30}$. Este hecho explica como una reacción peritumoral linfocítica marcada en el margen tumoral es un factor de buen pronóstico del CCR porque la reacción inflamatoria peritumoral puede promover la destrucción de las células de TB mediante un proceso de apoptosis ${ }^{31,32}$. La aplicación clínica deducida de estas investigaciones consiste en la posibilidad de desarrollar terapias inmunes que aumenten la reacción inflamatoria dirigida contra las células de tumor budding y mejorar el pronóstico de los pacientes ${ }^{33}$.

La falta de consenso en el método anatomopatológico que evalúa el grado de TB y su clasificación, así como la utilización de técnicas diferentes para identificar las células buds como la tinción con hematoxilina-eosina o técnicas inmunohistoquímica con citoqueratina dificultan la comparación de resultados en los diferentes trabajos. Sin embargo, a pesar de que el método para evaluar el grado de tumor budding no está estandarizado, todos los autores coinciden en que las categorías con mayor grado de $\mathrm{TB}$ se asocian a un peor pronóstico en el $\mathrm{CCR}^{14}$. En este estudio, se utiliza la tinción con hematoxilinaeosina y la clasificación descrita por Nakamura ${ }^{13}$. Es un método cualitativo con variaciones interobservador, aunque está validado y se incluye en la European Guidelines for Quality Assurance in Colorectal Screening and Diagnosis $1^{a} \mathrm{ed} .{ }^{12}$.

\section{Conclusión}

El Tumor budding de alto grado está asociado con otros factores de mal pronóstico del CCR como ganglios linfáticos regionales metastásicos, invasión linfática y perineural, patrón de crecimiento infiltrativo y depósitos tumorales discontinuos. El tumor budding de bajo grado se relaciona con otros factores de buen pronóstico del CCR con la reacción linfocítica peritumoral. El análisis del grado de TB podría ayudar a identificar pacientes con CCR de peor pronóstico.

\section{Referencias}

1. Jernel A, Bray F, Center MM. Global cancer statistics. CA Cancer J Clin. 2011;61:69-90.

2. Dukes CE. The relation of histology to spread in intestinal cancer. BR J Cancer 1950;4:59-62.

3. Edge SB, Byrd DR, Compton CC, Fritz AG, Green FL, Trotti A. AJCC Cancer Staging Manual. 7th ed. New York, Springer. 2010;143-64.

4. Compton C. Pronostic Factors in Cancer. 3rd ed. WileyLiss. New York. 2006.

5. Prall F. Tumour budding in colorectal carcinoma. Histopathology 2007;50:151-62.

6. Lugli A, Karamitopoulou E, Zlobec I. Tumour budding: a promising parameter in colorectal cancer. Br J Cancer 2012;106:1713-7.

7. Grizzi F, Celesti G, Basso G, Laghi L. Tumor budding as a potential histopathological biomarker in colorectal cancer: Hyde or hope? WJG 2012;18:6532-6.

8. Jiang B, Mason J, Jewett A, Qian J, Ding J, Cho W, et al. Cell budding from normal appearing epithelia: a predictor of colorectal cancer metastasis? Int J Biol Sci. 2013;9:119-33.

9. Hase K, Shatney C, Johnson D, Trollope M, Vierra M. Pronostic value of tumor budding in patients with colorectal cancer. Dis Colon Rectum 1993;36:627-35. 
10. Kye BH, Jung JH, Kim HJ, Kang SG, Cho HM, Kim G. Tumor budding as a risk factor of lymph node metastasis in submucosal invasive T1 colorectal carcinoma: a retrospective study. BMC Surgery 2012;12:16.

11. Ueno H, Murphy J, Jass JR, Mochizuki H. Tumour budding as an index to estimate the potential of aggressiveness in rectal cancer. Histopathology 2002;40:12732.

12. Quirke P, Risio M, Lambert Rm Karsa L, Viethm C. European Guidelines for Quality Assurance in Colorectal Screening and Diagnosis. Endoscopy. 2012;44:116-30.

13. Nakamura T, Mitomi H, Kikuchi S, Ohtami Y, Sato $\mathrm{K}$. Evaluation of the usefulness of tumor budding on the prediction of metastasis ti the lung and liver after curative excision of colorectal cancer. Hepatogastroenterology 2005;32:1432-5.

14. Puppa G, Senore C, Sheahan K, Vieth M, Luigli A, Zlobec I, et al. Diagnostic reproducibility of tumour budding in colorectal cancer: a multicentre, multinational study using virtual microscopy. Histopathology 2012;61:562-75.

15. Ohike N, Coban I, Kim GE. Tumor budding as a strong prognostic indicator in invasive ampullary adenocarcinomas. Am J Surg Pathol. 2010;34:1417-24.

16. Yamaguchi Y, Ishii G, Kojima M. Histopathologic features of the tumor budding in adenocarcinoma of the lung: tumor budding as an index to predict the potentional aggressiveness. J Thorac. Oncol. 2010;5:1361-8.

17. Choi HJ, Park KJ, Shin JS, Rob MS, Kwon HC, Lee HS. Tumor budding as a prognostic marker in stage III rectal carcinoma. Int J Colorectal Dis. 2007;22:863-8.

18. Nakamura T, Mitomi H, Kanazawa H, Ohkura Y, Watanabe M. Tumor budding as an index to identify high-risk patientes with stage II colon cancer. Dis Colon Rectum 2008;51:568-72.

19. Morodoni T, Isomoto H, Shirouzu K, Kakegawa K, Irie $\mathrm{K}$, Morimatsu M. An index for estimating the probability of lymph node metastasis in rectal cancers. Lymph node metastasis abd the histopathology of actively invasive regions of cancer. Cancer 1989;63:539-43.

20. Ogawa T, Yoshida T, Tsuruta T. Tumor budding is predictive of limphatic involvement ald lymph node metastases in submucosal invasive colorectal adenocarcinomas and in nonpolypoid compared with polypoid growths. Scnad J Gastroenterol. 2009;44:605-14.

21. Okuyama T, Oya M, Yamaguchi M. Budding (sprouting) as a useful prognostic marker in colorectal mucinous carcinoma. Jpn J Clin Oncol. 2002;32:412-6.
22. Gao P, Song Y, Wang Z, Xu Y, Tong L, Sun J, et al. Is the prediction of prognosis not improved by the seventh edition of the TNM classification for colorectal cancer? Analysis of the surveillance, epidemiology, and end results (SEER) database. BMC Cancer 2013;13:123-34.

23. Mroczkowski P, Schmidt U, Sahm M, Gastinger I, Lippert H, Kube R. Prognosis factors assessed for 15,096 patients with colon cancer in stages I and II. World J Surg. 2012;36:1693-8.

24. Schippinger W, Samonigg H, Scarberl-Moser R. A Prospective randomised phase III trial of adjuvant chemotherapy wirh 5-fluorouracil and leuvocorin in patients with stage II colon cancer. $\mathrm{Br}$ J Cancer 2007;97:1021-7.

25. Glimelius B, Cavalli-Björkman N. Metastatic colorectal cancer: current treatment and future options for improved survival. Medical approach present status. Scand J Gastroenterol. 2012;47:296-314.

26. Wang LM, Kevans D, Mulcahy H. Tumor budding is a strong and reproducible prognostic marker in T3N0 colorectal cancer. Am J Surg Pathol. 2009;33:134-41.

27. Mitrovic B, Schaeffer DF, Ridell RH, Kirsch R. Tumor budding in colorectal carcinoma: time to take notice. Mod Pathol. 2012;25:1315-25.

28. Giger O, Comtesse S, Lugli S, Zlobec I, Kurrer M. Intratumoral budding in preoperative biopsy specimens predicts lymph node and distant metastasis in patients with colorectal cancer. Dis Colon Rectum Mod Pathol. 2012;25:160-6.

29. Rogers AC, Gibbons D, Hanly A, Hyland J, O'Connell PR, Winter DC, et al. Prognosis significance of tumor budding in rectal cancer biopsies before neoadjuvant therapy. Mod Pathol. 2014;27:156-62. doi: 10.1038/ modpathol.2013.124. Epub 2013 Jul 26.

30. Shinto E, Mochizuki H, Ueno H, Matsubara O, Jass JR. A novel classification of tumor budding in colorectal cancer based on the presence of cytoplasmic pseudo-fragments around budding foci. Histopathology 2005; 47:25-31.

31. Jass JR, Love SB, Northover JM. A new prognosis classification of rectal cancer. Lancet 1987;I:1303-6.

32. Klintrup K, Makinen JM, Kauppila S, Vare PO, Melkko $\mathrm{J}$, Tuomiren H. Inflammation and prognosis in colorectal cancer. Eur J Cancer. 2005;41:2645-54.

33. Zlobec I, Lugli A, Baker K, Roth S, Mino P, Hatashi $\mathrm{S}$, et al. Role of APAF-I, E-cadherine and peritumoural lymphocytic infiltration in tumour budding in colorectal cancer. J Pathol. 2007;212:260-8. 